\title{
ON A CERTAIN CLASS OF LIMIT DISTRIBUTIONS AND THEIR DOMAIN OF ATTRACTION
}

\author{
BY \\ D. MEJZLER
}

1. Introduction. Let $\left\{\xi_{n}\right\}$ be a sequence of independent random variables having the same distribution function (d.f.) $F(x)$. We will consider the maximum term, i.e., the random variable

$$
\eta_{n}=\max \left(\xi_{1}, \xi_{2}, \cdots, \xi_{n}\right),
$$

whose d.f. $F_{n}(x)$ is

$$
F_{n}(x)=P\left\{\eta_{n}<x\right\}=F^{n}(x) .
$$

Much work has been done on the study of the limit distributions of the maximum term. The first results in this respect were obtained by M. Fréchet [1]. The most complete results, which may be said to summarize in a sense this series of investigations were obtained by B. V. Gnedenko [2]. He showed that the limit distributions for $F^{n}\left(a_{n} x+b_{n}\right)$, where $a_{n}>0$ and $b_{n}$ are suitably chosen real constants, are confined to the improper type and to the distributions of the following three forms:

$$
\begin{aligned}
\Lambda(x) & =\exp \left(-e^{-x}\right), \\
\Phi_{\alpha}(x) & = \begin{cases}0 & \text { if } x \leqq 0, \\
\exp \left(-x^{-\alpha}\right) & \text { if } x>0,\end{cases} \\
\Psi_{\alpha}(x) & = \begin{cases}\exp \left(-|x|^{\alpha}\right) & \text { if } x \leqq 0, \\
1 & \text { if } x>0,\end{cases}
\end{aligned}
$$

where $\alpha$ is a positive constant. Gnedenko also found the domain of attraction of the d.fs. $\Phi(x)$ from (1.1), i.e. the class of d.fs. $F(x)$ for which constants $a_{n}$ and $b_{n}$ may be found such that

$$
\lim _{n \rightarrow \infty} F^{n}\left(a_{n} x+b_{n}\right)=\Phi(x) .
$$

The problems involved in the theory of the limit distributions for the maximum term parallel those encountered in the theory of limit distributions for sums of

Received by the editors March 12, 1963. 
independent random variables. In the latter theory the following three classes of d.fs. play the central part [3]:

(a) The class of d.fs. which may be represented as limits of sums of independent and identically distributed summands. This class coincides with that of the stable laws.

(b) The class $L$ of d.fs. which may be represented as limits of sums of independent and "infinitesimal" summands.

(c) The class of d.fs. which may be represented as limits of subsequences of sums of independent and identically distributed summands. This class coincides with that of the infinitely divisible distributions.

Thus, in the theory of the maximum term, the class of d.fs. (1.1) is seen to be an analogue of the set of stable laws.

The analogue of the class $L$ was studied in [4]-[8]. Finally, it has been shown [9] that the analogue of the class of infinitely divisible distributions is the set of all distribution functions. In other words, for any d.f. $\Phi(x)$ there exists a d.f. $F(x)$ and integers $n_{k}$ and real numbers $a_{k}$ and $b_{k}$ such that

$$
\lim _{k \rightarrow \infty} F^{n} k\left(a_{k} x+b_{k}\right)=\Phi(x) .
$$

In connection with the last result, it would seem natural to restrict the problem by imposing certain conditions on the subsequence $\left\{n_{k}\right\}$ appearing in (1.2).

The d.f. $\Phi(x)$ will be called a partial limit of rank $r$, or $r$-limit, if there exists a d.f. $F(x)$, a subsequence of the positive integers, $\left\{n_{k}\right\}$, with

$$
\lim _{k \rightarrow \infty}\left(n_{k} / n_{k+1}\right)=r
$$

and constants $a_{k}$ and $b_{k}$ such that the d.f. of the random variable $\left(\eta_{n_{k}}-b_{k}\right) / a_{k}$ converges, as $k \rightarrow \infty$, to $\Phi(x)$, i.e. (1.2).

In this case $F(x)$ is said to be partially attracted with rank $r$ to the d.f. $\Phi(x)$, or $F(x)$ belongs to its $r$-attraction domain.

It is the purpose of this paper to study the $r$-limit distributions and their $r$ attraction domain $(r>0)$.

REMARK 1.1. The convergence of d.fs. is meant to be in the "weak sense," i.e., the functions converge at every continuity point of the limit function. Similarly, equality between two d.fs. (and even any two functions of bounded variation) will always mean that they coincide at their points of continuity.

In our considerations we shall often use the following two theorems:

Khintchine's Theorem ([10, TheOREM 43] OR [3, §10, TheOREM 1]). If the sequence $\left\{F_{n}(x)\right\}$ of $d$.fs. converges as $n \rightarrow \infty$ to a proper d.f. $F(x)$, then for any choice of the constants $a_{n}>0$ and $b_{n}$ the sequence $\left\{F_{n}\left(a_{n} x+b_{n}\right)\right\}$ can converge to a proper distribution only if this is of the same type as $F(x)$. However, for every sequence $\left\{F_{n}(x)\right\}$ of $d . f s$., there exist constants $a_{n}>0$ and $b_{n}$ such that $\left\{F_{n}\left(a_{n} x+b_{n}\right)\right\}$ converges to the improper distribution. 
Gnedenko's TheOREM [3, $\$ 10$, TheOREM 2]. For a sequence of d.fs. $\left\{F_{n}(x)\right\}$ the relations

$$
\begin{aligned}
& F_{n}\left(a_{n} x+b_{n}\right) \rightarrow F(x), \\
& F_{n}\left(\alpha_{n} x+\beta_{n}\right) \rightarrow F(x)
\end{aligned}
$$

as $n \rightarrow \infty$, where $a_{n}, \alpha_{n}, b_{n}, \beta_{n}$ are real constants and $F(x)$ is a proper d.f., are satisfied simultaneously if and only if

$$
\alpha_{n} / a_{n} \rightarrow 1, \quad\left(\beta_{n}-b_{n}\right) / a_{n} \rightarrow 0
$$

as $n \rightarrow \infty$.

2. Characterization of $r$-limit distributions. The characterization of $r$-limit distributions is given in [9]. The main results are formulated in Theorems 2.1-2.3.

THEOREM 2.1. (a) Every distribution is a 0-limit.

(b) The distribution $\Phi(x)$ is an $r$-limit $(0<r<1)$ if and only if $\Phi(x)$ and $\Phi^{r}(x)$ are of the same type; in other words, there exist constants $a=a(r)$ and $b=b(r)$ such that for every $x$

$$
\Phi^{r}(x)=\Phi(a x+b)
$$

(c) The distribution is a 1-limit if and only if it is an $r$-limit for every $0<r<1$.

The class of 1-limit distributions coincides with the class of limit distributions (1.1) for the maximum term.

Let $R(\Phi)$ denote the set of ranks $r$ for which $\Phi(x)$ is an $r$-limit. This set has a maximum $r_{0}=r_{0}(\Phi)$. Then umber $r_{0}$ will be called the maximal rank of partial attraction of the distribution $\Phi(x)$.

THEOREM 2.2. (a) $0<r_{0}<1$ if and only if

$$
R(\Phi)=\left\{r: r=r_{0}^{m}, m=1,2, \cdots, \infty\right\}
$$

(b) $r_{0}=1$ if and only if $R(\Phi)$ contains two numbers such that the ratio of their logarithms is irrational.

The "left end" $x_{0}=x_{0}(\Phi)$ and the "right end" $y_{0}=y_{0}(\Phi)$ of the distribution $\Phi(x)$ are defined by

$$
x_{0}=\operatorname{Inf}\{x: \Phi(x)>0\}, \quad y_{0}=\sup \{x: \Phi(x)<1\} .
$$

It can be shown that if a proper d.f. satisfies the condition (2.1) with $r>0$, then one, and only one, of the following cases must occur: 


$$
\begin{array}{lll}
a=1, & x_{0}=-\infty, & y_{0}=+\infty, \\
a>1, & x_{0}>-\infty, & y_{0}=+\infty, \\
a<1, & x_{0}=-\infty, & y_{0}<+\infty .
\end{array}
$$

Hence the class of distributions with partial attraction domain of positive rank is divided into three subclasses - to be denoted by $\Lambda^{*}, \Phi^{*}$ and $\Psi^{*}$, according to the cases (1), (2) and (3), respectively.

If $\Phi(x) \in \Phi^{*} \quad\left(\Phi(x) \in \Psi^{*}\right)$, then the distribution $\Phi_{0}(x)=\Phi\left(x+x_{0}\right)$ $\left(\Phi_{0}(x)=\Phi\left(x+y_{0}\right)\right)$ will satisfy a simpler functional relation

$$
\Phi_{0}^{r}(x)=\Phi_{0}(a x) .
$$

The distribution $\Phi(x)$ is continuous at $x_{0}\left(y_{0}\right)$, hence the function $\Phi\left(e^{x}+x_{0}\right)$ $\left(\Phi\left(-e^{-x}+y_{0}\right)\right)$ is a distribution function.

THEOREM 2.3. $\Phi(x) \in \Phi^{*}\left(\Phi(x) \in \Psi^{*}\right)$ if and only if

$$
\Phi\left(e^{x}+x_{0}\right) \in \Lambda^{*}\left(\Phi\left(-e^{-x}+y_{0}\right) \in \Lambda^{*}\right) .
$$

It is easy to find a canonical representation of these distributions. Using the last theorem it is enough to study the class $\Lambda^{*}$.

THEOREM 2.4. The function $\Phi(x)$ is a d.f. of the class $\Lambda^{*}$ if and only if for every $x$

$$
0<\Phi(x)<1
$$

and its iterated logarithm is represented by

$$
\lg |\lg \Phi(x)|=\phi(x)-\alpha x,
$$

where $\alpha$ is a positive constant and $\phi(x)$ is a bounded periodic (in the sense of Remark 1.1) function satisfying

$$
\phi\left(x_{2}\right)-\phi\left(x_{1}\right) \leqq \alpha\left(x_{2}-x_{1}\right)
$$

for every $x_{1}<x_{2}$.

If the d.f. $\Phi(x)$ is represented in this form and $b_{0}$ is the period of $\phi(x)$, then the maximal rank $r_{0}$ of $\Phi(x)$ is given by

$$
r_{0}=\exp \left(-\alpha b_{0}\right)
$$

and we have for every $x$

$$
\Phi^{r_{0}}(x)=\Phi\left(x+b_{0}\right) \text {. }
$$

In particular, if $\phi(x)$ is a constant, then $\Phi(x)$ belongs to the type $\Lambda(x)(1.1)$ and (2.10) holds for every $r$ and $b$ satisfying (2.9).

Proof. Let $\Phi(x) \in \Lambda^{*}$ and let $r_{0}<1$. From equalities (2.1) and (2.4) follow inequalities (2.6) and there exists a $b_{0}>0$ such that (2.10) becomes an identity. 
The function $f(x)=\lg |\lg \Phi(x)|$ is defined because of (2.6) and satisfies the conditions

$$
f(-\infty)=+\infty, f(+\infty)=-\infty,
$$

(2) $\quad f(x)$ nonincreasing function,

$$
f\left(x+b_{0}\right)-f(x)=\lg r_{0} .
$$

Let

$$
\alpha=\left(-\lg r_{0}\right) / b_{0}, \quad \phi(x)=\alpha x+f(x),
$$

then we obtain (2.7). From (2.11) it follows that $\phi\left(x+b_{0}\right)-\phi(x)=0$. It is easy to see that $b_{0}$ is the minimal period of $\phi(x)$ : If for some integer $n>1$ $\phi\left(x+b_{0} / n\right)-\phi(x)=0$, then by (2.12) we get for every $x$

$$
\Phi^{r_{0}^{1 / n}}(x)=\Phi\left(x+b_{0} / n\right),
$$

which contradicts the definition of $r_{0}$.

The necessity of (2.8) follows from the monotonicity of $f(x)$. The proof of the sufficiency of these conditions is analogous. Equalities (2.9) and (2.10) are evident. Let us remark that since every integral multiple of the period $b_{0}$ is again a period of $\phi(x)$ and by (2.9) $r_{0}^{n}=\exp \left(-\alpha n b_{0}\right)$ for every integer $n$, therefore (2.10) implies

$$
\Phi^{r 0^{n}}(x)=\Phi\left(x+n b_{0}\right)
$$

for every $x$ and integer $n$.

COROllaRY 2.1. A differentiable d.f. $\Phi(x)$ belongs to $\Lambda^{*}$ if and only if the derivative of $\lg |\lg \Phi(x)|$ is periodic.

In order to avoid condition (2.8) let us state the following

Corollary 2.2. The function $\Phi(x)$ is a d.f. of the class $\Lambda^{*}$ if and only if it satisfies (2.6) and for every $x$

$$
\lg |\lg \Phi(x)|=g(x-b[x / b])+[x / b] \lg r
$$

( $[x]$ is the integral part of $x$ ), where $0<r<1$ and $b>0$ are constants and $g(x)$ is nonincreasing and bounded in the interval $[0, b)$ and satisfies

$$
g(b-0)-g(0+) \geqq \lg r .
$$

As an immediate consequence of Theorems 2.3 and 2.4 we obtain

THEOREM 2.5. The distribution $\Phi(x)$ belongs to $\Lambda^{*}, \Phi^{*}, \Psi^{*}$ if and only if it has the form 


$$
\begin{array}{rlr}
\Phi(x) & =\{\Lambda(\alpha x)\}^{P(x)}, & \\
\Phi\left(x+x_{0}\right) & =\left\{\Phi_{\alpha}(x)\right\}^{P(\lg x)} & (x>0), \\
\Phi\left(x+y_{0}\right) & =\left\{\Psi_{\alpha}(x)\right\}^{P(\cdot \lg |x|)} & (x<0),
\end{array}
$$

respectively, where $\alpha$ is a positive constant, $P(x)$ a positive periodic function of bounded variation, the numbers $x_{0}, y_{0}$ are the "ends" of the distribution $\Phi(x)$ and $\Lambda(x), \Phi_{\alpha}(x), \Psi_{x}(x)$ are distributions (1.1).

3. A generalization of the problem. It might seem that the definition of $r$-limit distributions $(r>0)$ is artificial and that it would be more natural to replace (1.3) by the weaker assumption

$$
\underset{k \rightarrow \infty}{\lim _{i n f}}\left(n_{k} / n_{k+1}\right)>0 \text {. }
$$

We shall show that this extends neither the class of $r$-limit distributions nor their domain of partial attraction.

LEMMA 3.1. Let $\Phi(x)$ be a proper distribution satisfying (1.2), where (3.1) holds. If for some subsequence $k(s)$ the limit

$$
\lim _{s \rightarrow \infty}\left(n_{k(s)} / n_{k(s)+1}\right)=r
$$

exists, where $0<r \leqq 1$, then the following limits exist

$$
\lim _{s \rightarrow \infty}\left(a_{k(s)+1} / a_{k(s)}\right)=a, \quad \lim _{s \rightarrow \infty}\left(\left(b_{k(s)+1}-b_{k(s)}\right) / a_{k(s)}\right)=b
$$

and (2.1) holds.

Proof. We have

$$
\begin{aligned}
\lim _{s \rightarrow \infty} F^{n_{k(s)}}\left(a_{k(s)} x+b_{k(s)}\right) & =\Phi(x) \text { and } \\
\lim _{s \rightarrow \infty} F^{n_{k(s)+1}}\left(a_{k(s)+1} x+b_{k(s)+1}\right) & =\Phi(x) .
\end{aligned}
$$

Hence, because of (3.2), also

$$
\lim _{s \rightarrow \infty} F^{n_{k(s)}}\left(a_{k(s)+1} x+b_{k(s)+1}\right)=\Phi^{r}(x) .
$$

In view of. Khintchine's theorem this relation together with (3.3) implies the necessity of (2.1). The last equality can be put in the form

$$
\lim _{s \rightarrow \infty} F^{n_{k(s)}}\left(a_{k(s)+1} x+b_{k(s)+1}\right)=\Phi(a x+b) .
$$

If we substitute $(x-b) / a$ for $x$ in the last equality, the proof then follows by (3.3) and Gnedenko's theorem. 
THEOREM 3.1. If conditions (1.2) and (3.1) hold, then:

(a) The distribution $\Phi(x)$ has a partial domain of attraction of positive rank.

(b) The d.f. $F(x)$ is partially attracted to $\Phi(x)$ with every rank $r$ in $R(\Phi)$.

Proof. Let

$$
\liminf _{k \rightarrow \infty}\left(n_{k} / n_{k+1}\right)=r \quad(0<r<1) .
$$

(a) According to Khintchine's theorem, every improper distribution is an $r$-limit for every $0 \leqq r \leqq 1$, hence the first part of the theorem follows from Lemma 3.1.

(b) Let us consider two cases.

I. Let $0<r_{0}<1$. By Lemma 3.1, Theorem 2.2 and equality (3.4) we conclude that every partial limit of the ratio $n_{k} / n_{k+1}$ which is smaller than 1 has to have the form (2.2). Therefore there exists an integer $n$ such that $r=r_{0}^{n}$ and the set of partial limits of the sequence $\left\{n_{k} / n_{k+1}\right\}$ does not include any other number except

$$
1, r_{0}, r_{0}^{2}, \cdots, r_{0}^{n}=r \text {. }
$$

Let us first show that there is a subsequence $\left\{n_{k(s)}\right\}$ with

$$
r_{0}^{n}=\liminf _{s \rightarrow \infty}\left(n_{k(s)} / n_{k(s+1)}\right) \leqq \limsup _{s \rightarrow \infty}\left(n_{k(s)} / n_{k(s+1)}\right) \leqq r_{0} .
$$

Using (3.4) we can assume that for every $k$

$$
\left(n_{k} / n_{k+1}\right) \geqq r_{0}^{n+1 / 4} \text {. }
$$

Let $q$,

$$
r_{0}^{n+1 / 4}<q<1
$$

be given. Take $n_{k(1)}=n_{1}, n_{k(s+1)}=n_{k(s)+p}$, where

$$
p=\min \left\{m: n_{k(s)} \leqq q \cdot n_{k(s)+m}\right\} .
$$

Then from (3.7) and (3.8) it follows that

$$
\begin{aligned}
q \geqq\left(n_{k(s)} / n_{k(s+1)}\right) & =\left(n_{k(s)} / n_{k(s+1)-1}\right) \cdot\left(n_{k(s+1)-1} / n_{k(s+1)}\right) \\
& >q \cdot r_{0}^{n+1 / 4}>r_{0}^{n+1 / 2} .
\end{aligned}
$$

Condition (1.2) is satisfied also for the subsequence $\left\{n_{k(s)}\right\}$, hence the partial limits of the ratio $n_{k(s)} / n_{k(s+1)}$ are again of the form (2.2). On the other hand it is clear that

$$
\liminf _{s \rightarrow \infty}\left(n_{k(s)} / n_{k(s+1)}\right) \leqq \limsup _{k \rightarrow \infty}\left(n_{k} / n_{k+1}\right)
$$

This together with (3.5) yields (3.6). In order to simplify notation we may assume that the original sequence $\left\{n_{k}\right\}$ satisfies, for every $k$, the inequalities 


$$
r_{0}^{n+1 / 2}<\left(n_{k} / n_{k+1}\right)<r_{0}^{1 / 2}
$$

Consider the intervals

$$
\Delta_{p}=\left[r_{0}^{p+1 / 2}, r_{0}^{p-1 / 2}\right), \quad p=1,2, \cdots, n .
$$

From (3.9) we have for every integer $k$ a $p(k)$ such that

$$
\left(n_{k} / n_{k+1}\right) \in \Delta_{p(k)} \text {. }
$$

Let the integers $n_{k, s}$ be introduced between $n_{k}$ and $n_{k+1}$, where

$$
n_{k, s}=\left[n_{k} / r_{0}^{s}\right] \quad(s=1,2, \cdots, p(k)-1) .
$$

By assumption there exist $a_{0}$ and $b_{0}$ such that for every $x$

$$
\Phi^{r_{0}}(x)=\Phi\left(a_{0} x+b_{0}\right) \text {. }
$$

Let us also supplement the sequences $\left\{a_{k}\right\}$ and $\left\{b_{k}\right\}$, by adding the numbers

$$
\begin{aligned}
a_{k, s}=a_{k} \cdot a_{0}^{s}, \quad b_{k, s}=b_{k}+b_{0}\left(1+a_{0}+\cdots\right. & \left.+a_{0}^{s-1}\right) a_{k}, \\
& (s=1,2, \cdots, p(k)-1) .
\end{aligned}
$$

The supplemented sequences will be denoted by $\left\{\bar{n}_{k}\right\},\left\{\bar{a}_{k}\right\}$ and $\left\{\bar{b}_{k}\right\}$, respectively. Let us prove that

$$
\lim _{k \rightarrow \infty} F^{\bar{n}_{k}}\left(\bar{a}_{k} x+\bar{b}_{k}\right)=\Phi(x) .
$$

It is enough to show that if for a certain subsequence $k(t)$

$$
\lim _{t \rightarrow \infty} F^{\bar{n}_{k(t)}}\left(\bar{a}_{k(t)} x+\bar{b}_{k(t)}\right)=f(x),
$$

then

$$
f(x)=\Phi(x)
$$

Using (3.11) and (3.13) we can represent $\bar{n}_{k(t)}, \bar{a}_{k(t)}$ and $\bar{b}_{k(t)}$ in the form

$$
\begin{aligned}
& \bar{n}_{k(t)}=\left[n_{k(t)} / r^{s(t)}\right], \quad \bar{a}_{k(t)}=a_{0}^{s(t)} a_{k(t)}, \\
& \bar{b}_{k(t)}=b_{k(t)}+b_{0}\left(1+a_{0}+\cdots+a_{0}^{s(t)-1}\right) a_{k(t)}, \\
& \left(\bar{b}_{k(t)}=b_{k(t)} \text { if } s(t)=0\right),
\end{aligned}
$$

where $0 \leqq s(t) \leqq p(k)-1 \leqq n-1$.

Without restricting the generality of our argument we may assume that $s(t)=m$, where $m$ is a constant non-negative integer. For $m=0$ the sequence (3.15) is a subsequence of (1.2) and equality (3.16) is certainly satisfied. Let $m \geqq 1$; then, by (3.17), the equality (3.15) can be restated as 


$$
\lim _{t \rightarrow \infty}\left[F\left(a_{k(t)}\left(a_{0}^{m} x+b_{0}\left(1+a_{0}+\cdots+a_{0}^{m-1}\right)\right)+b_{k(t)}\right)\right]^{\left[n_{k(t)} / r_{0}^{m}\right]}=f(x),
$$

hence, from (1.2), we get

$$
f^{r_{0}^{m}}(x)=\Phi\left(a_{0}^{m} x+b_{0}\left(1+a_{0}+\cdots+a_{0}^{m-1}\right)\right) .
$$

By iterating relation (3.12) $m$ times we get

$$
\Phi^{r_{0}^{m}}(x)=\Phi\left(a_{0}^{m} x+b_{0}\left(1+a_{0}+\cdots+a_{0}^{m-1}\right)\right),
$$

which is valid for every integer $m$. From this follows (3.16) and the proof of (3.14). Let us now prove that

$$
\lim _{k \rightarrow \infty}\left(\bar{n}_{k} / \bar{n}_{k+1}\right)=r_{0}
$$

Let $\bar{n}_{k}$ and $\bar{n}_{k+1}$ be two successive terms in the sequence. We have the following cases:

(1) $\left(\bar{n}_{k} / \bar{n}_{k+1}\right)=\left(n_{k, s} / n_{k, s+1}\right)$, where $0 \leqq s \leqq p(k)-1$. Then

$$
\begin{aligned}
r_{0}\left(n_{k}-1\right) / n_{k} & <r_{0}\left(n_{k}-r_{0}^{s}\right) / n_{k}<n_{k, s} / n_{k, s+1} \\
& <r_{0} n_{k} /\left(n_{k}-r_{0}^{s+1}\right)<r_{0} n_{k} /\left(n_{k}-1\right) .
\end{aligned}
$$

(2) $\left(\bar{n}_{k} / \bar{n}_{k+1}\right)=\left(n_{k, p(k)-1} / n_{k+1}\right)$. Then

$$
\left(n_{k} / n_{k+1}\right) r_{0}^{1-p(k)}-\left(1 / n_{k+1}\right)<\left(n_{k, p(k)-1} / n_{k+1}\right) \leqq\left(n_{k} / n_{k+1}\right) r_{0}^{1-p(k)} \text {. }
$$

But by (3.10), $r_{0}^{p(k)+1 / 2} \leqq\left(n_{k} / n_{k+1}\right)<r_{0}^{p(k)-1 / 2}$, therefore

$$
r_{0}^{3 / 2}-\left(r_{0} / n_{k+1}\right)<\left(n_{k, p(k)-1} / n_{k+1}\right)<r_{0}^{1 / 2} \text {. }
$$

(3) If both $\bar{n}_{k}$ and $\bar{n}_{k+1}$ belong to the original sequence $n_{k}$, then

$$
r_{0}^{3 / 2} \leqq\left(\bar{n}_{k} / \bar{n}_{k+1}\right)<r_{0}^{1 / 2},
$$

since in this case $p(k)=1$.

From (3.19), (3.20) and (3.21) it follows that

$$
r_{0}^{3 / 2} \leqq \liminf _{k \rightarrow \infty}\left(\bar{n}_{k} / \bar{n}_{k+1}\right) \leqq \limsup _{k \rightarrow \infty}\left(\bar{n}_{k} / \bar{n}_{k+1}\right) \leqq r_{0}^{1 / 2}
$$

In view of (3.14) the partial limits of the ratio $\bar{n}_{k} / \bar{n}_{k+1}$ are also of the form (3.5). Thus the last inequalities imply (3.18).

Let $m$ be any integer and suppose $n_{k}^{\prime}=n_{k m+c}$, where $c$ is a non-negative integer. From (3.18) it follows that $\lim _{k \rightarrow \infty}\left(n_{k}^{\prime} / n_{k+1}^{\prime}\right)=r_{0}^{m}$, and by Theorem 2.2 we get the proof of the present theorem for the first case.

REMARK 3.1. We have also proved, incidentally, that if (1.2) and (3.1) hold then there exists a sequence of powers $\left\{n_{k}^{\prime}\right\}$ such that (1.2) is satisfied and 


$$
\liminf _{k \rightarrow \infty}\left(n_{k} / n_{k+1}\right)=\lim _{k \rightarrow \infty}\left(n_{k}^{\prime} / n_{k+1}^{\prime}\right)=r .
$$

This does not imply that $\left\{n_{k}^{\prime}\right\}$ is a subsequence of $\left\{n_{k}\right\}$.

II. Let $r_{0}=1$. By Khintchine's theorem every distribution $F(x)$ belongs to the domain of attraction of the improper type. Hence we may assume that $\Phi(x)$ is one of the distributions (1.1). Denote

$$
\begin{aligned}
& n_{k, s}=n_{k}\left(n_{k+1} / n_{k}\right)^{s / k}, \quad a_{k, s}=a_{k}\left(a_{k+1} / a_{k}\right)^{s / k} \\
& b_{k, s}=b_{k}+\left(b_{k+1}-b_{k}\right) s / k, \quad s=0,1, \cdots, k-1 ; \quad k=1,2, \cdots .
\end{aligned}
$$

By (3.1) we get $\lim \sup _{k \rightarrow \infty}\left(n_{k+1} / n_{k}\right)<\infty$, hence

$$
\lim _{k \rightarrow \infty}\left(n_{k, s} / n_{k, s+1}\right)=1
$$

(where $n_{k, k}=n_{k+1,0}$ ). Since $n_{k, s}<n_{k, s+1}<n_{k+1,0}$ and $\lim _{k \rightarrow \infty} n_{k, s}=\infty$, every integer $n\left(n \geqq n_{1}\right)$ can be (uniquely) represented as $n=n_{k, s}+m$, where $0 \leqq m=m(n)<n_{k, s+1}-n_{k, s}$ and $\lim _{k \rightarrow \infty}\left(m / n_{k, s}\right)=0$ by (3.22). Suppose $a_{n}=a_{k, s}, b_{n}=b_{k, s}$, then we shall prove that

$$
\lim _{n \rightarrow \infty} F^{n}\left(a_{n} x+b_{n}\right)=\Phi(x) .
$$

Indeed, suppose that for a certain subsequence $n(t)$

$$
\lim _{t \rightarrow \infty} F^{n(t)}\left(a_{n(t)} x+b_{n(t)}\right)=f(x)
$$

where $n(t)=n_{k(t)}\left(n_{k(t)+1} / n_{k(t)}\right)^{s(t) / k(t)}+m(t)$.

Using Lemma 3.1 and condition (1.2) the following limits may be assumed to exist:

and

$$
\lim _{t \rightarrow \infty}\left(a_{k(t)+1} / a_{k(t)}\right)=a, \quad \lim _{t \rightarrow \infty}\left(\left(b_{k(t)+1}-b_{k(t)}\right) / a_{k(t)}\right)=b
$$

$$
\lim _{t \rightarrow \infty}\left(n_{k(t)} / n_{k(t)+1}\right)=r \quad(r>0) .
$$

Now, since $0 \leqq s / k<1$, we may assume the existence of $\lim _{t \rightarrow \infty}(s(t) / k(t))=c$, where $0 \leqq c \leqq 1$. Hence

$$
\begin{array}{ll}
a_{n(t)}=a_{k(t)}\left(a^{c}+o(1)\right), & b_{n(t)}=b_{k(t)}=a_{k(t)}(b c+o(1)), \\
n(t)=n_{k(t)}\left(r^{-c}+o(1)\right), & \text { as } t \rightarrow \infty .
\end{array}
$$

Thus (3.24) takes the form

$$
\lim _{t \rightarrow \infty}\left\{F\left(a_{k(t)}\left(a^{c} x+b c+\alpha_{t}(x)\right)+b_{k(t)}\right)\right\}_{k(t)}^{n}{ }^{\left(r^{-c}+\beta_{t}\right)}=f(x),
$$

where $\alpha_{t}(x)=o(1), \beta_{t}=o(1)$ as $t \rightarrow \infty$. Combined with (1.2) this yields 


$$
f^{r^{c}}(x)=\Phi\left(a^{c} x+b c\right) .
$$

Equality (2.1) is satisfied by Lemma 3.1. It is easy to see that if $\Phi(x)$ is one of the distributions (1.1) then, together with (2.1), it also satisfies

$$
\Phi^{r c}(x)=\Phi\left(a^{c} x+b c\right)
$$

for every real $c$. Thus we get $f(x)=\Phi(x)$, which proves (3.23). Clearly, for every $0 \leqq r<1$ it is possible to extract from (3.23) a subsequence which satisfies condition (1.3). This proves the proposition in the second case.

REMARK 3.2. Let $\Phi(x)$ be an arbitrary distribution. To each d.f. $F(x)$ we let correspond the set $N(\Phi, F)$ of all increasing sequences of positive integers $\left\{n_{k}\right\}$ which satisfy (1.3) and for which there exist constants $a_{k}$ and $b_{k}$ such that (1.2) holds. Put

$$
E(\Phi)=\bigcup_{F} N(\Phi, F),
$$

where $F$ runs through the class of all d.fs.

Now let $\bar{N}(\Phi, F)$ be the set of all $\left\{n_{k}\right\}$, for which (1.2) is satisfiable, and let

$$
\bar{E}(\Phi)=\bigcup_{F} \bar{N}(\Phi, F) .
$$

Evidently $N \subset \bar{N}$. The Theorem 3.1 can be rephrased as follows:

$$
\begin{aligned}
\left\{r: r=\liminf _{k \rightarrow \infty}\left(n_{k} / n_{k+1}\right),\left\{n_{k}\right\}\right. & \in \bar{N}(\Phi, F)\} \\
= & \left\{r: r=\lim _{k \rightarrow \infty}\left(n_{k} / n_{k+1}\right),\left\{n_{k}\right\} \in N(\Phi, F)\right\}
\end{aligned}
$$

for any two d.fs. $\Phi(x)$ and $F(x)$. In particular, if $\Phi(x)$ is an $r$-limit distribution $(r>0)$ and the d.f. $F(x)$ is partially attracted to $\Phi(x)$ with a positive rank, then also

$$
\begin{aligned}
\left\{r: r=\liminf _{k \rightarrow \infty}\left(n_{k} / n_{k+1}\right),\left\{n_{k}\right\}\right. & \in \bar{E}(\Phi)\} \\
& =\left\{r: r=\lim _{k \rightarrow \infty}\left(n_{k} / n_{k+1}\right),\left\{n_{k}\right\} \in N(\Phi, F)\right\} .
\end{aligned}
$$

In [9] it was noted that each $r$-limit distribution $(r>0)$ belongs to its own domain of $r$-attraction. Denoting by $N(\Phi, \Phi)$ the set of all sequences $\left\{n_{k}\right\}$ for which the limit (1.3) exists and

$$
\lim _{k \rightarrow \infty} \Phi^{n_{k}}\left(a_{k} x+b_{k}\right)=\Phi(x)
$$

is satisfiable, we obtain the following result:

$$
\begin{aligned}
\left\{r: r=\liminf _{k \rightarrow \infty}\left(n_{k} / n_{k+1}\right),\left\{n_{k}\right\}\right. & \in \bar{E}(\Phi)\} \\
& =\left\{r: r=\lim _{k \rightarrow \infty}\left(n_{k} / n_{k+1}\right),\left\{n_{k}\right\} \in N(\Phi, \Phi)\right\}
\end{aligned}
$$

for every $r$-limit distribution $\Phi(x)(r>0)$. 
Thus conditions (1.2) and (3.1) and conditions (3.25) and (1.3), where $r>0$, determine the same set of limit distributions.

The maximal rank of partial attraction of the distribution $\Phi(x)$ was defined as

$$
r_{0}(\Phi)=\sup _{E(\Phi)} \lim _{k \rightarrow \infty}\left(n_{k} / n_{k+1}\right)
$$

(In virtue of Theorem 2.1a, $E(\Phi)$ is not empty.) It follows from our considerations that this definition may be replaced by

$$
r_{0}(\Phi)=\sup _{\bar{E}(\Phi)} \liminf _{k \rightarrow \infty}\left(n_{k} / n_{k+1}\right)
$$

and also by

$$
r_{0}(\Phi)= \begin{cases}0 & \text { if } N(\Phi, \Phi) \text { is empty, } \\ \sup _{N(\Phi, \Phi)} \lim _{k \rightarrow \infty}\left(n_{k} / n_{k+1}\right) \text { otherwise. }\end{cases}
$$

The set $N(\Phi, \Phi)$ may actually be empty. In [9] it was shown that the distribution

$$
H(x)=\left\{\begin{array}{lr}
0 \quad \text { if } x \leqq 0, \\
1-1 / \lg (x+e)
\end{array} \quad \text { if } x>0\right.
$$

cannot be partially attracted to any proper distribution : for every $\Phi(x)$ the set $\bar{N}(\Phi, H)$ is empty and therefore so is $N(H, H)$. (This example was given by $\mathrm{A}$. Khintchine $[10, \S 15]$ in connection with an analogous problem concerning sums of random independent variables.)

It seems plausible that the condition " $N(\Phi, \Phi)$ nonempty" is not only necessary but also sufficient in order that $\Phi(x)$ be an $r$-limit with $r>0$.

4. A comparison between $r$-attraction domains for $r>0$ and $r=0$. The notion of distribution type, which is essential in the theory of limit distributions for sums of independent random variables, has to be extended for our purposes.

Definition 4.1. The distributions $\Phi(x)$ and $G(x)$ belong to the same distribution "family," if for some real constants $a>0, b$ and $c>0$ and every $x$

$$
G(x)=\Phi^{c}(a x+b) \text {. }
$$

It is easy to see that together with every distribution $\Phi(x)$ not only all distributions of the same type but also every distribution of the same family is a limit for the maximum term, normalized in an appropriate way. This is true also for the domain of attraction.

The family is called proper if it contains a proper d.f.

THEOREM 4.1. A d.f. can be partially attracted with a positive rank to at most one proper family. 
Proof. Assume that (1.2) and (1.3) hold and also

$$
\lim _{k \rightarrow \infty} F^{v_{k}}\left(\alpha_{k} x+\beta_{k}\right)=G(x),
$$

where $\Phi(x)$ and $G(x)$ are proper distributions. To every integer $k$ (such that $\left.v_{k} \geqq n_{1}\right)$ there corresponds an integer $m=m(k)$ such that

$$
n_{m} \leqq v_{k}<n_{m+1} \text {. }
$$

By (1.3) the ratio $v_{k} / n_{m}$ is bounded. Let (4.2) be written in the form

$$
\lim _{k \rightarrow \infty}\left\{F^{n_{m}}\left(\alpha_{k} x+\beta_{k}\right)\right\}^{v_{k} / n_{m}}=G(x) .
$$

Let us extract a subsequence $k_{s}$ so that the following limits exist:

$$
\lim _{s \rightarrow \infty}\left\{F\left(\alpha_{k_{s}} x+\beta_{k_{s}}\right)\right\}^{n_{m}(k s)} \text { and } \lim _{s \rightarrow \infty}\left(v_{k_{s}} / n_{m\left(k_{s}\right)}\right) .
$$

Then (4.1) follows from Khintchine's theorem and condition (1.2).

Theorem 3.1 implies that for a given distribution $\Phi(x)$ it is meaningful to speak about two domains of partial attraction only: those with "zero density" and those with "positive density.",

THEOREM 4.2. The 0-attraction domain of a distribution is effectively larger than its attraction domain of positive rank.

Proof. Let us first suppose that $\Phi(x) \in \Lambda^{*}$. By Theorem 2.4,

$$
\Phi(x)=\exp \left(-e^{\phi(x)-\alpha x}\right),
$$

where $\alpha>0$ is a constant and $\phi(x)$ is a bounded periodic function satisfying condition (2.8). Without restricting the generality of the argument we may assume that the period $b_{0}$ of $\phi(x)$ is 1 .

Let $g(x)$ be another bounded and periodic function with the same period 1 , which also satisfies (2.8). Then the distribution

$$
G(x)=\exp \left(-e^{g(x)-\alpha x}\right)
$$

too, belongs to $\Lambda^{*}$. We may choose $g(x)$ so that the distributions $\Phi(x)$ and $G(x)$ will not be of the same family and so that they will have the same value and the same one-sided limits at the points 0 and 1 . Thus the function

$$
F(x)= \begin{cases}0 & \text { if } x \leqq 0 \\ \Phi(x) & \text { if } 2 n(n-1)<x \leqq 2 n^{2} \\ G(x) & \text { if } 2 n^{2}<x \leqq 2 n(n+1)\end{cases}
$$

$$
(n=1,2, \cdots)
$$


is a d.f. Let $r_{0}=\exp (-\alpha)$. Because of (2.9), $\Phi(x)$ and $G(x)$ satisfy condition (2.13), where $b_{0}=1$. Let

$$
b_{k}=2 k^{2}-k, \quad n_{k}=\left[r_{0}^{-b_{k}}\right]
$$

Then

$$
\lim F^{n_{k}}\left(x+b_{k}\right)=\Phi(x) .
$$

Indeed, let $x$ be any number. If $[|x|]<k$, then $2 k(k-1)<x+b_{k}<2 k^{2}$, hence by (4.3), from some $k$ on $F\left(x+b_{k}\right)=\Phi\left(x+b_{k}\right)$. But $b_{k}$ is an integer, hence by (2.13), from some $k$ on we will have

$$
F\left(x+b_{k}\right)=\Phi^{r_{0}^{b_{k}}}(x)
$$

hence

$$
F^{n_{k}}\left(x+b_{k}\right)=\{\Phi(x)\}^{n_{k} r_{0}^{b_{k}}} .
$$

From the definition (4.4) of $n_{k}$ it follows that

$$
\left(r_{0}^{-b_{k}}-1\right) r_{0}^{b_{k}}<n_{k} r_{0}^{b_{k}} \leqq 1,
$$

and since $0<r_{0}<1, \lim _{k \rightarrow \infty}\left(n_{k} r_{0}^{b_{k}}\right)=1$. This and equality (4.6) prove (4.5). Thus we have proved that $F(x)$ belongs to the domain of partial attraction of $\Phi(x)$. If we assume

$$
b_{k}=2 k^{2}+k, \quad n_{k}=\left[r_{0}^{-b_{k}}\right],
$$

then a similar argument shows that $F(x)$ is partially attracted also to the law $G(x)$. But $G(x)$ was constructed so that it does not belong to the same family as $\Phi(x)$ and by the previous theorem the d.f. $F(x)$ cannot be attracted to $\Phi(x)$ with positive rank.

In order to avoid using the canonical representation of distributions in $\Phi^{*}$ or $\Psi^{*}$, we use the fact that the d.f. $F(x)$ constructed above is partially attracted to the distribution $\Phi(x)$ in such a way that the constants $a_{k}$ in (1.2) are equal to 1 . Also, for every $x$,

$$
F(x)<1 \text {. }
$$

Now let $T(x)$ be any distribution in $\Phi^{*}$. Let us assume

$$
\Phi(x)=T\left(e^{x}+x_{0}\right),
$$

where $x_{0}$ is the left end of $T(x)$, which is defined by (2.3). By Theorem 2.3, $\Phi(x) \in \Lambda^{*}$. Let $F(x), n_{k}$ and $b_{k}$ be defined by (4.3) and (4.4). Take

$$
t(x)= \begin{cases}0 & \text { if } x \leqq 0 \\ F(\lg x) & \text { if } x>0\end{cases}
$$


By (4.8), $t(x)$ is a d.f. For every $x>0$ we get from (4.5)

$$
\lim _{k \rightarrow \infty} F^{n_{k}}\left(\lg x+b_{k}\right)=\Phi(\lg x) .
$$

Let $a_{k}=\exp \left(b_{k}\right)$, then from (4.9), (4.10) and (4.11) it follows that for every $x>0$

$$
\lim _{k \rightarrow \infty} t^{n_{k}}\left(a_{k} x\right)=T\left(x+x_{0}\right)
$$

On the other hand, for $x \leqq 0$

$$
t\left(a_{k} x\right)=T\left(x+x_{0}\right)=0,
$$

thus (4.12) is satisfied for every $x$, or $t(x)$ is partially attracted to $T(x)$. Let us take the sequences (4.7), a similar argument proves that

$$
\lim _{k \rightarrow \infty} t^{n_{k}}\left(a_{k} x\right)=W\left(x+x_{0}\right)
$$

where $G(x)=W\left(e^{x}+x_{0}\right)$. It is easy to see that if $\Phi(x)$ and $G(x)$ are not of the same family neither are $T(x)$ and $W(x)$, which proves our statement for distributions in the class $\Phi^{*}$. The proof for distributions in $\Psi^{*}$ is analogous. Here we take

$$
\begin{aligned}
& \Phi(x)=T\left(-e^{-x}+y_{0}\right), \quad a_{k}=\exp \left(-b_{k}\right), \\
& t(x)= \begin{cases}F(-\lg |x|) & \text { if } x<0, \\
1 & \text { if } x \geqq 0 .\end{cases}
\end{aligned}
$$

EXAMPLE 4.1. Let

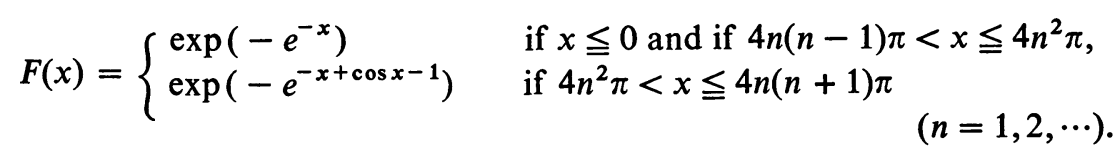

It is easy to verify that $F(x)$ is a d.f. If

$$
b_{k}=\left(4 k^{2}-2 k\right) \pi, \quad n_{k}=\left[\exp \left(b_{k}\right)\right]
$$

then

$$
\lim _{k \rightarrow \infty} F^{n_{k}}\left(x+b_{k}\right)=\Lambda(x) .
$$

But if we take $b_{k}=\left(4 k^{2}+2 k\right) \pi, n_{k}=\left[\exp \left(b_{k}\right)\right]$, then

$$
\lim _{k \rightarrow \infty} F^{n_{k}}\left(x+b_{k}\right)=\exp \left(-e^{-x+\cos x}\right) \text {. }
$$

Notice that the latter distribution is also in $\Lambda^{*}$, since it satisfies

$$
\Phi^{e^{2 \pi}}(x+2 \pi)=\Phi(x) \text {. }
$$


Using Theorem 4.2 we can conclude that $F(x)$ cannot be partially attracted with a positive rank to any proper distribution.

EXAMPLE 4.2. Let

$$
F(x)=\left\{\begin{array}{ll}
\exp \left(-e^{-x}\right) & \text { if } x \leqq 0 \text { and if } 2 n(n-1)<x \leqq 2 n^{2}, \\
\exp \left(-e^{-x^{2} / 2 n+2 n x-2 n^{3}-2 n^{2}}\right) & \text { if } 2 n^{2}<x \leqq 2 n(n+1)
\end{array} \quad(n=1,2, \cdots) .\right.
$$

This d.f. is also not partially attracted with positive rank to any proper distribution, since we have (4.13), when $b_{k}=2 k^{2}-k, n_{k}=\left[\exp \left(b_{k}\right)\right]$ and

$$
\lim _{k \rightarrow \infty} F^{n_{k}}\left(a_{k} x+b_{k}\right)=\Phi(x),
$$

where $a_{k}=\sqrt{ }(2 k), b_{k}=2 k^{2}, n_{k}=\left[\exp \left(b_{k}\right)\right]$,

$$
\Phi(x)= \begin{cases}0 & \text { if } x<0, \\ \exp \left(-e^{-x^{2}}\right) & \text { if } x \geqq 0 .\end{cases}
$$

Notice that the distribution $\Phi(x)$ is not an $r$-limit for $r>0$.

5. Uniqueness of the numerical sequences. Let us see to what degree the sequences $\left\{n_{k}\right\},\left\{a_{k}\right\}$ and $\left\{b_{k}\right\}$ are determined by the functions $\Phi(x)$ and $F(x)$ and conditions (1.2) and (1.3).

THEOREM 5.1. Assume the validity of (1.2) and

$$
\lim _{k \rightarrow \infty}\left(n_{k} / n_{k+1}\right)=r_{0}
$$

where $r_{0}\left(0<r_{0}<1\right)$ is the maximal rank of the distribution $\Phi(x)$. Assume also that

$$
\lim _{k \rightarrow \infty} F^{v_{k}}\left(\alpha_{k} x+\beta_{k}\right)=\Phi(x)
$$

where

$$
\limsup _{k \rightarrow \infty}\left(v_{k} / v_{k+1}\right)<1
$$

\section{Then}

(a) There exists an integer-valued function on the indices $k \mu=\mu(k)$ so that for sufficiently large $k$

$$
\mu(k+1)>\mu(k)
$$

and 


$$
\begin{aligned}
& \lim _{k \rightarrow \infty}\left(v_{k} / n_{\mu(k)}\right)=1, \quad \lim _{k \rightarrow \infty}\left(\alpha_{k} / a_{\mu(k)}\right)=1, \\
& \left.\lim _{k \rightarrow \infty}\left(\beta_{k}-b_{\mu(k)}\right) / a_{\mu(k)}\right)=0 .
\end{aligned}
$$

(b) In particular if we have also

$$
\lim _{k \rightarrow \infty}\left(v_{k} / v_{k+1}\right)=r_{0},
$$

then there exists an integer $p$ such that

$$
\begin{array}{ll}
\lim _{k \rightarrow \infty}\left(v_{k} / n_{k+p}\right)=1, & \lim _{k \rightarrow \infty}\left(\alpha_{k} / a_{k+p}\right)=1, \\
\lim _{k \rightarrow \infty}\left(\left(\beta_{k}-b_{k+p}\right) / a_{k+p}\right)= & 0 .
\end{array}
$$

Proof. (a) To sufficiently large $k$ 's $\left(v_{k} \geqq n_{1}\right)$ there correspond integers $m=m(k)$ defined by

Put

$$
n_{m} \leqq v_{k}<n_{m+1}
$$

$$
\mu(k)= \begin{cases}m & \text { if } v_{k} \leqq\left(n_{m} / n_{m+1}\right)^{1 / 2} \\ m+1 & \text { otherwise }\end{cases}
$$

hence

$$
\left(n_{m} / n_{m+1}\right)^{1 / 2}<\left(v_{k} / n_{\mu(k)}\right) \leqq\left(n_{m+1} / n_{m}\right)^{1 / 2} .
$$

If for some subsequence $k_{s}$ the limit

$$
\lim _{s \rightarrow \infty}\left(v_{k_{s}} / n_{\mu\left(k_{e}\right)}\right)=c
$$

exists, then from above inequalities and (5.1) it follows that

$$
r_{0}^{1 / 2} \leqq c<r_{0}^{-1 / 2}
$$

Using (1.2) and (5.2), we deduce

$$
\lim _{s \rightarrow \infty} F^{\mu\left(k_{s}\right)}\left(\alpha_{k_{s}} x+\beta_{k_{s}}\right)=\Phi^{1 / c}(x)
$$

and

$$
\lim _{s \rightarrow \infty} F^{\left(k_{s}\right)}\left(a_{\mu\left(k_{s}\right)} x+b_{\mu\left(k_{s}\right)}\right)=\Phi(x) .
$$

Thus by Khintchine's theorem the d.fs. $\Phi(x)$ and $\Phi^{1 / c}(x)$ belong to the same type. From Theorem 2.2 it follows that $c=r_{0}^{m}$, where $m$ is an integer. This and (5.8) imply (5.5). Using the first of these equalities together with (5.3) we get

$$
\liminf _{k \rightarrow \infty}\left(n_{\mu(k+1)} / n_{\mu(k)}\right)>r_{0}^{-1}>1 \text {, }
$$

which proves (5.4). 
(b) The first of the equalities (5.5) together with (5.6) implies that

$$
\lim _{k \rightarrow \infty}\left(n_{\mu(k)} / n_{\mu(k+1)}\right)=r_{0} .
$$

Hence by (5.1) we conclude that for large $k$ 's

$$
\mu(k+1)-\mu(k)=1,
$$

or for these $k$ 's

$$
\mu(k)=p+k
$$

where $p$ is an integer. Therefore the relations (5.5) take the form (5.7).

In order to explain the content of this theorem let us call two triples of numerical sequences $\left(n_{k}, a_{k}, b_{k}\right)$ and $\left(v_{k}, \alpha_{k}, \beta_{k}\right)$ asymptotically equivalent if

$$
\lim _{k \rightarrow \infty}\left(v_{k} / n_{k}\right)=1, \quad \lim _{k \rightarrow \infty}\left(\alpha_{k} / a_{k}\right)=1, \quad \lim _{k \rightarrow \infty}\left(\left(\beta_{k}-b_{k}\right) / a_{k}\right)=0,
$$

since only in this case equality (1.2) remains valid when we replace one triple by the other. This implies that together with (5.2) we have also

$$
\lim _{k \rightarrow \infty} F^{v_{k}+t_{k}}\left(\alpha_{k} x+\beta_{k}\right)=\Phi(x)
$$

if $t_{k}=o\left(v_{k}\right)$ as $k \rightarrow \infty$. Therefore every sequence of the form (5.2) may contain "superfluous" terms, the presence of which does not influence its "density" or the value of the lower limit (3.1). Thus the first part of the theorem can be rephrased as follows: Every sequence of the form (5.2) which does not contain "superfluous" terms (condition (5.3)) is asymptotically equivalent to a certain subsequence of (1.2), if this sequence has the maximal "density" $r_{0}$.

Now, the sequences $\left\{n_{k+p}\right\},\left\{a_{k+p}\right\},\left\{b_{k+p}\right\}$ are translations of the original sequences $\left\{n_{k}\right\},\left\{a_{k}\right\},\left\{b_{k}\right\}$, hence the second part of the theorem can be rephrased to read:

The representation of the distribution $\Phi(x)$ by the d.f. $F(x)$ in the form (1.2) with the additional condition (5.1) is unique in the following sense: the functions $\Phi(x)$ and $F(x)$ and the conditions (1.2) and (5.1) determine the sequences $\left\{n_{k}\right\}$, $\left\{a_{k}\right\},\left\{b_{k}\right\}$ "asymptotically" up to a translation.

A slightly more general form of the second half of the previous theorem is:

COROLlary 5.1. Assume (1.2) and (5.2) and that the following limits exist and are positive

$$
\lim _{k \rightarrow \infty}\left(n_{k} / n_{k+1}\right)=r, \quad \lim _{k \rightarrow \infty}\left(v_{k} / v_{k+1}\right)=\rho .
$$

Let $r_{0} \quad\left(0<r_{0}<1\right)$ be the maximal rank of $\Phi(x)$. Put

$$
m=\left(\lg r_{0}\right) / \lg r, \quad \mu=\left(\lg r_{0}\right) / \lg \rho .
$$


Then $m$ and $\mu$ are integers and there exists an integer $p$ such that

$$
\begin{aligned}
\lim _{k \rightarrow \infty}\left(v_{m k} / n_{\mu k}\right) & =r_{0}^{-p}, \quad \lim _{k \rightarrow \infty}\left(\alpha_{m k} / a_{\mu k}\right)=a_{0}^{p}, \\
\left.\lim _{k \rightarrow \infty}\left(\beta_{m k}-b_{\mu k}\right) / a_{\mu k}\right) & =b_{0}\left(1+a_{0}+\cdots+a_{0}^{p-1}\right),
\end{aligned}
$$

where $a_{0}, b_{0}$ are defined by (3.12).

In particular, if $r=\rho=r_{0}$, then these equalities coincide with (5.7).

6. A special case of attraction to distributions of the class $\Lambda^{*}$. Let $\Phi(x)$ be an $r$-limit distribution $(r>0)$. Our aim is to characterize its $r$-attraction domain. By Theorem 3.1, if $\Phi(x)$ is one of the distributions (1.1), then its attraction domain of positive rank is equal to its full attraction domain, which is characterized in [2] and [11]. Hence it is enough to study the case when $\Phi(x)$ is not one of the distributions (1.1).

Theorem 5.1 suggests that the characterization of the $r$-attraction domain can be given by indicating a method of construction of the numerical sequences $\left\{n_{k}\right\},\left\{a_{k}\right\}$ and $\left\{b_{k}\right\}$ with the aid of the given $\Phi(x)$ and $F(x)$. The conditions found by us will in fact be formulated in these terms.

We give only a partial solution of the problem. For $\Phi(x) \in \Lambda^{*}$ we consider only a special case of attraction, namely, when the constants $a_{k}$ appearing in (1.2) are equal to 1 , i.e., instead of (1.2) we assume

$$
\lim _{k \rightarrow \infty} F^{n_{k}}\left(x+b_{k}\right)=\Phi(x)
$$

In addition we shall assume that the limit distribution $\Phi(x)$ is continuous and the d.f. $F(x)$ is continuous for sufficiently large $x$.

In the next section we shall see that the general case (1.2) with respect to the distributions of classes $\Phi^{*}$ and $\Psi^{*}$ may be reduced to the special case (6.1) here considered.

We start with some lemmas. Throughout these lemmas we assume (6.1) and (5.1), where

$$
0<r_{0}<1 \text {. }
$$

Let $b_{0}$ be the number given in (2.10) and let $\eta\left(0<\eta<b_{0}\right)$ be any number. Put

$$
\phi(x)=\frac{\lg \Phi(x+\eta)}{\lg \Phi(x)} .
$$

By (2.6), $\phi(x)$ is defined and continuous for every $x$. It is also periodic with period $b_{0}$ and of bounded variation in every final interval. Let $M$ and $m$ be the maximum and minimum of $\phi(x)$. By (6.2), $\Phi(x) \neq \Lambda(x)$, hence $m<M$. A theorem of S. Banach [12, Chapter VIII, §5, Corollary 2, p. 227] implies that for almost every 
value $y$ in $[m, M]$ the equation $y=f(x)$ has a finite number of solutions in every finite interval. Let us choose a number $A$ with

$$
m<A<M
$$

and let $0 \leqq \gamma_{1}<\gamma_{2}<\cdots<\gamma_{n}<b_{0}$ be all the solutions of equation

$$
\phi(x)=A
$$

in the interval $\left[0, b_{0}\right)$. Put

$$
\tau=\min _{1 \leqq i \leqq n}\left(\gamma_{i+1}-\gamma_{i}\right) \quad\left(\gamma_{n+1}=b_{0}+\gamma_{1}\right) .
$$

LEMMA 6.1. For every finite $x$ we have

$$
F(x)<1 \text {. }
$$

Proof. Since in our case (6.1) $a_{k} \equiv 1$ we deduce from Lemma 3.1 that

$$
\lim _{k \rightarrow \infty}\left(b_{k+1}-b_{k}\right)=b_{0} \text {. }
$$

Hence it is clear that $b_{k} \rightarrow \infty$. If there existed an $L$ such that $F(x)=1$ for $x>L$ then, for all $x$ and sufficiently large $k, F\left(x+b_{k}\right)=1$. Hence by (6.1) also $\Phi(x) \equiv 1$ which is impossible.

Let $F(x)$ be continuous and positive for $x \geqq T$. By (6.7) the function

$$
f(x)=\frac{\lg F(x+\eta)}{\lg F(x)},
$$

where the number $\eta$ was given in (6.3), is defined and continuous for $x \geqq T$. We may assume that the number $A$ in (6.4) and (6.5) is such that the solutions of equation

$$
f(x)=A
$$

have no accumulation point. By (6.1) we have for every $x$

$$
\lim _{k \rightarrow \infty} f\left(x+b_{k}\right)=\phi(x) \text {. }
$$

LEMMA 6.2. Let $\varepsilon$ and $\delta(0<\varepsilon<\delta<\tau)$ be given numbers. There exists a number $B=B(\varepsilon, \delta)$ such that for every root $x^{\prime}>B$ of $(6.10)$

$$
f(x) \neq 0 \text { provided } \varepsilon<\left|x-x^{\prime}\right|<\delta \text {. }
$$

Proof. It is enough to show that $f(x) \neq 0$ for $x^{\prime}+\varepsilon<x<x^{\prime}+\delta$. Assume that this is not true. Then there exist $\varepsilon_{0}$ and $\delta_{0}\left(0<\varepsilon_{0}<\delta_{0}<\tau\right)$ and two sequences $\left\{x_{k}\right\}$ and $\left\{x_{k}^{\prime}\right\}$ of roots of (6.10) such that for every $k$

$$
x_{k}+\varepsilon_{0}<x_{k}^{\prime}<x_{k}+\delta_{0}
$$


and $x_{k} \rightarrow \infty$ as $k \rightarrow \infty$. To every $x_{k}$ we let correspond the term $b_{m(k)}$ from the sequence $\left\{b_{k}\right\}$ which is closest to $x_{k}$ from the left. Hence

Put

$$
b_{m(k)} \leqq x_{k}<b_{m(k)+1} \text {. }
$$

$$
x_{k}=b_{m(k)}+t_{k}, x_{k}^{\prime}=b_{m(k)}+t_{k}^{\prime}
$$

then

$$
0 \leqq t_{k}<b_{m(k)+i}-b_{m(k)}
$$

and by (6.8) the sequence $\left\{t_{k}\right\}$ is bounded. Also by (6.12) and (6.13),

$$
0<\varepsilon_{0} \leqq t_{k}^{\prime}-t_{k} \leqq \delta_{0}<\tau .
$$

Without restricting the generality of our argument we may assume the existence of the limits $\lim _{k \rightarrow \infty} t_{k}=t$ and $\lim _{k \rightarrow \infty} t_{k}^{\prime}=t^{\prime}$. Thus

$$
0<t^{\prime}-t<\tau \text {. }
$$

On the other hand, from (6.10) and (6.11) and the definition of $\left\{x_{k}\right\}$ and $\left\{x_{k}^{\prime}\right\}$ it follows that

$$
A=f\left(x_{k}\right)=f\left(t_{k}+b_{m(k)}\right)=\phi(t)
$$

and

$$
A=f\left(x_{k}^{\prime}\right)=f\left(t_{k}^{\prime}+b_{m(k)}\right)=\phi\left(t^{\prime}\right) .
$$

Thus $t$ and $t^{\prime}$ are solutions of (6.5), which contradicts (6.14) by the definition of $\tau$ in (6.6).

DefinITION 6.1. The root $\xi$, respectively $\bar{x}$, of the equation (6.5), respectively (6.10), is said to be a "distinguished" root (a d-root) if

$$
\{\phi(\xi+\tau / 2)-A\} \cdot\{\phi(\xi-\tau / 2)-A\}<0,
$$

respectively

$$
\{f(\bar{x}+\tau / 2)-A\} \cdot\{f(\bar{x}-\tau / 2)-A\}<0,
$$

where $\tau$ is given by (6.6). The existence of d-roots of equation (6.5) is guaranteed by (6.4).

Definition 6.2. The two sequences $\left\{\alpha_{k}\right\}$ and $\left\{\alpha_{k}^{\prime}\right\}$ are said to be equivalent, $\alpha_{k} \sim \alpha_{k}^{\prime}$, if

$$
\lim _{k \rightarrow \infty}\left(\alpha_{k}-\alpha_{k}^{\prime}\right)=0 \text {. }
$$

LEMMA 6.3. For every $d$-root $\xi$ of (6.5) there exists a sequence $\{\bar{x}(k)\}$ of $d$-roots of (6.10) such that

$$
b_{k} \sim \bar{x}(k)-\xi
$$


Proof. Let $\xi$ be a d-root of (6.5). Let us assume for definiteness that for all sufficiently small positive $x, \phi(x+\xi)>A$. By (6.11), for every sufficiently small $\varepsilon>0$,

$$
\lim _{k \rightarrow \infty} f\left(\xi+\varepsilon+b_{k}\right)=\phi(\xi+\varepsilon)>A
$$

and

$$
\lim _{k \rightarrow \infty} f\left(\xi-\varepsilon+b_{k}\right)=\phi(\xi-\varepsilon)<A .
$$

Hence from some $k$ on

$$
f\left(\xi-\varepsilon+b_{k}\right)<A, \quad f\left(\xi+\varepsilon+b_{k}\right)>A .
$$

Therefore for sufficiently large $k$ there is in every one of the intervals

$$
\left(\xi-\varepsilon+b_{k}, \xi+\varepsilon+b_{k}\right)
$$

a solution to (6.10). But according to Lemma 6.2 the intervals

$$
\left[\xi+b_{k}-\delta+\varepsilon, \xi+b_{k}-2 \varepsilon\right]
$$

and

$$
\left[\xi+b_{k}+2 \varepsilon, \xi+b_{k}+\delta-\varepsilon\right]
$$

do not contain any roots of (6.10) if $k$ is sufficiently large. Hence by (6.17), $f(x)<A$ if $x$ is in (6.18) while $f(x)>A$ if $x$ is in (6.18'). If initially $\varepsilon$ is sufficiently small $(0<\varepsilon<\tau / 6)$ and $\delta$ is close enough to $\tau(5 \tau / 6<\delta<\tau)$ then, for every $x$ in (6.17), $x-\tau / 2$ is in (6.18) and $x+\tau / 2$ in (6.18'). Hence all the roots of (6.10) in the interval (6.17) are d-roots. Let us define $\bar{x}(k)$ as the d-root which is closest to $b_{k}+\xi$. The definition is legitimate since these roots do not have an accumulation point. Now, for large $k$ 's, $\bar{x}(k)$ is in (6.17), hence $\left|\bar{x}(k)-b_{k}-\xi\right|<\varepsilon$, which proves (6.15).

COROLlaRY 6.1. The set of d-roots of (6.10) is unbounded.

Let us define a sequence $\{t(n)\}$ of d-roots of (6.10) as follows: $t(1)$ will be arbitrary and

$$
t(n+1)=\min \{\bar{x}: \bar{x} \geqq t(n)+\tau / 2\} .
$$

LEMMA 6.4. For any sequence $\{\bar{x}(k)\}$ of d-roots of equation (6.10) that satisfies

$$
\lim _{k \rightarrow \infty} \inf (\bar{x}(k+1)-\bar{x}(k))>0
$$

there exists an integer $p$ and a subsequence $\left\{n_{k}\right\}$ such that

$$
t\left(n_{k}\right) \sim \bar{x}(p+k) .
$$


Proof. From (6.20) it follows that $\bar{x}(k) \rightarrow \infty$. By Lemma 6.2 we have $\lim \inf _{k \rightarrow \infty}(\bar{x}(k+1)-\bar{x}(k)) \geqq \tau$. Let $p$ be sufficiently large so that

$$
\bar{x}(p) \geqq t(1), \quad \bar{x}(k+1)-\bar{x}(k)>\tau / 2 \quad \text { for } k \geqq p .
$$

To $\bar{x}(p+k)$ corresponds the term $t\left(n_{k}\right)$ of the sequence $\{t(n)\}$ which is closest to it from the left. According to (6.19) such a term exists, hence

$$
t\left(n_{k}\right) \leqq \bar{x}(p+k)<t\left(n_{k}+1\right) .
$$

It is easy to see that this correspondence defines a subsequence, i.e., $n(k+1)>n(k)$ (if we allow repetitions the lemma holds even without assuming (6.20)). Indeed, from (6.22) it follows that

$$
\bar{x}(p+k+1)>\bar{x}(p+k)+\tau / 2 \geqq t\left(n_{k}\right)+\tau / 2,
$$

hence, by (6.19), $t\left(n_{k+1}\right) \leqq \bar{x}(p+k+1)$. But according to (6.23) this means that $n(k)<n(k)+1 \leqq n(k+1)$. In order to prove (6.21) suppose that there exist an $\varepsilon_{0}>0$ and $t\left(n_{k_{s}}\right), \bar{x}\left(p+k_{s}\right)$ such that, for every natural $s, x\left(p+k_{s}\right)-t\left(n_{k_{s}}\right) \geqq \varepsilon_{0}$. By Lemma 6.2, $\bar{x}\left(p+k_{s}\right)-t\left(n_{k_{s}}\right)>\tau / 2$ for sufficiently large $s$. Hence by (6.19) also $\bar{x}\left(p+k_{s}\right) \geqq t\left(n_{k_{s}+1}\right)$, which contradicts the definition of $n_{k}$ in (6.23).

COROLlaRY 6.2. There exists a subsequence $\left\{n_{k}\right\}$ and a d-root $\xi$ of equation (6.5) such that

$$
b_{k} \sim t\left(n_{k}\right)-\xi
$$

Proof. According to Lemmas 6.3, 6.4 and condition (6.8) we have $b_{p+k} \sim t\left(n_{k}\right)-\xi^{\prime}$, where $p$ is an integer, $\xi^{\prime}$ a d-root of (6.5) and $\left\{n_{k}\right\}$ is a certain subsequence. But by (6.8), $b_{p+k} \sim b_{k}+p \cdot b_{0}$, hence $b_{k} \sim t\left(n_{k}\right)-\left(\xi^{\prime}+p b_{0}\right)$. Since $b_{0}$ is the period of $\phi(x),\left(\xi^{\prime}+p b_{0}\right)$ is also a d-root of $(6.5)$.

LEMMA 6.5. Assume (6.24). If $\xi^{\prime}$ is the d-root of (6.5) following $\xi$ then

$$
b_{k} \sim t\left(n_{k}+1\right)-\xi^{\prime}
$$

Proof. Let

$$
\xi^{\prime}-\xi=\tau^{\prime}
$$

From (6.11) and (6.24) follows

$$
\lim _{k \rightarrow \infty} f\left(t\left(n_{k}\right)+\tau^{\prime} \pm \varepsilon\right)=\phi\left(\xi^{\prime} \pm \varepsilon\right)
$$

Since $\xi^{\prime}$ is a d-root of (6.5), for every $\varepsilon>0$ and for all large $k$ the intervals

$$
\left(t\left(n_{k}\right)+\tau^{\prime}-\varepsilon, t\left(n_{k}\right)+\tau^{\prime}+\varepsilon\right)
$$

contain a root of (6.10) each. Using the same argument as in the proof of Lemma 
6.3, it can be seen that for sufficiently large $k$ all the roots that are in (6.27) are d-roots. Now, if we prove that, from some $k$ on, the intervals

$$
\left(t\left(n_{k}\right)+\tau / 2, t\left(n_{k}\right)+\tau^{\prime}-\varepsilon\right)
$$

do not contain d-roots, it will follow from the definition of the sequence $\{t(n)\}$ in (6.19) that

$$
t\left(n_{k}+1\right) \in\left(t\left(n_{k}\right)+\tau^{\prime}-\varepsilon, t\left(n_{k}\right)+\tau^{\prime}+\varepsilon\right),
$$

which proves (6.25) by (6.24) and (6.26). Moreover, it is easy to see that in (6.28) there are no roots of $(6.10)$. Indeed, suppose that for some $\varepsilon_{0}>0$, a subsequence $\left\{n\left(k_{s}\right)\right\}$ and a sequence $\left\{x_{s}\right\}$ of roots of $(6.10)$,

$$
t\left(n_{k_{s}}\right)+\tau / 2<x_{s} \leqq t\left(n_{k_{s}}\right)+\tau^{\prime}-\varepsilon_{0}
$$

for every $s$. Let

$$
x_{s}=t\left(n_{k_{s}}\right)+z_{s},
$$

then $\tau / 2<z_{s} \leqq \tau^{\prime}-\varepsilon_{0}$. We may assume that $\lim _{s \rightarrow \infty} z_{s}=z$. Then

$$
0<z<\tau^{\prime} .
$$

By (6.24) and (6.29), $x_{s} \sim \xi+z+b_{k_{s}}$. But since $x_{s}$ is a root of (6.10) we have, by (6.11), $\phi(\xi+z)=A$. Therefore $(\xi+z)$ is again a root of (6.5). This contradicts the definition of $\xi^{\prime}$ since, by $(6.30), \xi<\xi+z<\xi^{\prime}$.

For our purpose it is enough to give a method to construct a sequence $\left\{\beta_{k}\right\}$ for which

$$
\lim _{k \rightarrow \infty}\left(\beta_{k}-b_{k}\right)
$$

exists and is finite.

LEMMA 6.6. Let $m$ be the number of d-roots of $(6.5)$ in $\left[0, b_{0}\right)$. In order that for the sequence of indices $\left\{n_{k}\right\}$ the finite limit

$$
\lim _{k \rightarrow \infty}\left(t\left(n_{k}\right)-b_{k}\right)
$$

exist, it is necessary and sufficient that for large $k$ this sequence coincides with an arithmetic sequence $\left\{v_{k}\right\}$ of the form

$$
v_{k}=c+m(k-1),
$$

where $c$ is an integer.

If this limit exists then it is a d-root of (6.5).

Proof. Let $\xi$ be the limit (6.32). By (6.11) using Gnedenko's theorem we get

$$
\lim _{k \rightarrow \infty} f\left(x+t\left(n_{k}\right)-\xi\right)=\phi(x),
$$


hence, substituting $x=\xi, x=\xi-\tau / 2, x=\xi+\tau / 2$, we establish that $\xi$ is a d-root of (6.5). This proves the second part of our lemma.

Now assume (6.24). By using the previous lemma $m$ times we get

$$
b_{k} \sim t\left(n_{k}+m\right)-\xi_{m},
$$

where $\xi_{m}$ is the $m$ th next root to $\xi$. Since $\xi_{m}=b_{0}+\xi$, then, from (6.24), $b_{k}+b_{0} \sim t\left(n_{k}+m\right)-\xi$ and, by (6.8), $b_{k+1} \sim t\left(n_{k}+m\right)-\xi$. On the other hand, by (6.24) also $b_{k+1} \sim t\left(n_{k+1}\right)-\xi$, therefore

$$
t\left(n_{k+1}\right) \sim t\left(n_{k}+m\right)
$$

By (6.19), for every $n, t(n+1)-t(n) \geqq \tau / 2>0$, hence (6.34) implies that for sufficiently large $k, t\left(n_{k+1}\right)=t\left(n_{k}+m\right)$. Thus also $n_{k+1}=n_{k}+m$ for large $k$. This means that for sufficiently large $k$ the sequence $\left\{n_{k}\right\}$ coincides with a certain $\left\{v_{k}\right\}$ of the form (6.33), which proves the necessity of our condition.

By Corollary 6.2, there exist a sequence of indices $\left\{n_{k}\right\}$ and a d-root $\xi$ of equation (6.5) so that (6.24) holds. Because of the necessity of our condition, $n_{k}$ has the form (6.33) for large $k$. But it is easy to see that if the limit (6.32) exists for some sequence which has the form (6.33) for large $k$ then, by the previous lemma and (6.8), it exists also for every sequence that has the form (6.33) from some $k$ on. Thus we have proved that our condition is sufficient.

Corollary 6.3.

$$
\lim _{n \rightarrow \infty}(t(n+m)-t(n))=b_{0}
$$

Proof. Consider the $m$ subsequences $t_{k, c}$,

$$
t_{k, c}=t(c+m(k-1)), \quad c=1,2, \cdots, m .
$$

According to the lemma and condition (6.8), for every $\varepsilon>0$ there is an $N$ so that if $k \geqq N$ then $\left|t_{k+1, c}-t_{k, c}-b_{0}\right|<\varepsilon$ uniformly in $c(1 \leqq c \leqq m)$. On the other hand, for every $n$ there are a $c$ and a $k$ such that $t(n)=t_{k, c}$ and thus $t(n+m)=t_{k+1, c}$. Therefore, for $n>m N$ we have $\left|t(n+m)-t(n)-b_{0}\right|<\varepsilon$.

With the same notation as before let us construct another sequence $\left\{\beta_{k}\right\}$ which satisfies condition (6.31).

Lemma 6.7. Let $\beta_{1}\left(\beta_{1}>T\right)$ be any number. Define

$$
\beta_{k+1}=\min \left\{\bar{x}: \bar{x} \geqq \beta_{k}+b_{0}-\tau / 2\right\} .
$$

Then the limit (6.31) exists and is a d-root of (6.5).

Proof. By Lemma 6.4 there exists a sequence $\left\{n_{k}\right\}$ and an integer $p \geqq 0$ such that

$$
t\left(n_{k}\right) \sim \beta_{p+k}
$$


But Corollary 6.3 implies that $t\left(n_{k}+m\right) \sim t\left(n_{k}\right)+b_{0}$. Hence, by (6.36), $t\left(n_{k}+m\right) \sim \beta_{p+k}+b_{0}$, which means that for every $\varepsilon(0<\varepsilon<\tau / 2)$

$$
\beta_{p+k}+b_{0}-\varepsilon<t\left(n_{k}+m\right)<\beta_{p+k}+b_{0}+\varepsilon
$$

for all large $k$. Now by Lemma 6.2 and (6.19)

$$
t\left(n_{k}+m\right)-t\left(n_{k}+m-1\right)>\tau-\varepsilon
$$

for all large $k$. Hence, by (6.37), for all large $k$ equation (6.10) does not have solutions in the interval

$$
\left(\beta_{p+k}+b_{0}-\tau / 2, \beta_{p+k}+b_{0}-\varepsilon\right)
$$

and

$$
\min \left\{\bar{x}: \bar{x} \geqq \beta_{p+k}+b_{0}-\tau / 2\right\}=\min \left\{\bar{x}: \bar{x} \geqq t\left(n_{k}+m-1\right)+\tau / 2\right\} .
$$

This together with (6.19) and (6.35) implies that, for all large $k, t\left(n_{k}+m\right)=\beta_{p+k+1}$. But from (6.36) we get $t\left(n_{k+1}\right) \sim \beta_{p+k+1}$, therefore, from some $k$ on, $t\left(n_{k+1}\right) \sim t\left(n_{k}+m\right)$. By the previous lemma this is enough to guarantee the existence of the limit (6.32). Let this limit be $\xi$. Then by (6.36) also

$$
\lim _{k \rightarrow \infty}\left(\beta_{p+k}-b_{k}\right)=0,
$$

which, together with (6.8), implies the existence of the limit (6.31) and that this is equal to $\left(\xi+p b_{0}\right)$. But since $\xi$ is a d-root of $(6.5),\left(\xi+p b_{0}\right)$ is also a d-root.

THEOREM 6.1. Let $\Phi(x)$ be a continuous distribution of the class $\Lambda^{*}$ with the maximal rank $r_{0}\left(0<r_{0}<1\right)$. Let $F(x)$ be a d.f. which is continuous from some $x$ on.

In order that there exist sequences of real numbers $\left\{b_{k}\right\}$ and positive integers $\left\{n_{k}\right\}$, satisfying condition (5.1), so that (6.1) holds, the following conditions are necessary and sufficient:

$\left(^{*}\right) F(x)<1$ for every $x$;

(**) $\lim _{k \rightarrow \infty}\left(\beta_{k+1}-\beta_{k}\right)=b_{0}$, where $\left\{\beta_{k}\right\}$ is the sequence (6.35) and $b_{0}$ is the parameter in (2.10);

$\left({ }^{* * *}\right) \lim _{k \rightarrow \infty}\left(\lg F\left(x+\beta_{k}\right) / \lg F\left(\beta_{k}\right)\right)=\lg \Phi(x+\xi) / \lg \Phi(\xi)$, where $\xi$ is a d-root of equation (6.5) in $\left[0, b_{0}\right)$.

Proof. I. Necessity. Assume (5.1) and (6.1). The necessity of (*) is proved in Lemma 6.1. Under the assumptions of the theorem, relations (6.8) and (6.31), and thus condition (**), hold. Let $\xi^{\prime}$ be the limit of (6.31). By (6.38) and Gnedenko's theorem we have for every $x$

$$
\lim _{k \rightarrow \infty} \frac{\lg F\left(x+\beta_{k}\right)}{\lg F\left(\beta_{k}\right)}=\frac{\lg \Phi\left(x+\xi^{\prime}\right)}{\lg \Phi\left(\xi^{\prime}\right)} .
$$


As was proved, $\xi^{\prime}$ is a d-root of (6.5). But the distribution $\Phi(x)$ belongs to $\Lambda^{*}$, hence it satisfies condition (2.9). Hence for every $x$ and every integer $n$

$$
\frac{\lg \Phi\left(x+\xi^{\prime}+n b_{0}\right)}{\lg \Phi\left(\xi^{\prime}+n b_{0}\right)}=\frac{\lg \Phi\left(x+\xi^{\prime}\right)}{\lg \Phi\left(\xi^{\prime}\right)}
$$

which proves that the number $\xi$ in condition $\left({ }^{* * *}\right)$ can be taken in the interval $\left[0, b_{0}\right)$.

II. Sufficiency. Assume that the d.f. $F(x)$ satisfies conditions $\left({ }^{*}\right),\left({ }^{* *}\right)$ and $\left({ }^{* * *}\right)$. Let

$$
b_{k}=\beta_{k}-\xi, \quad n_{k}=\left[(\lg \Phi(\xi)) / \lg F\left(\beta_{k}\right)\right] .
$$

By $\left({ }^{*}\right)$, the sequence $\left\{n_{k}\right\}$ is defined for every $k$. Since, according to $\left({ }^{* *}\right), \beta_{k} \rightarrow \infty$ then also $n_{k} \rightarrow \infty$ and $b_{k} \rightarrow \infty$ as $k \rightarrow \infty$. For $x-\xi$ instead of $x$, condition (***) takes the form

$$
\lim _{k \rightarrow \infty}\left\{F\left(x+b_{k}\right)\right\}^{(\lg \Phi(\xi)) / \lg F\left(\beta_{k}\right)}=\Phi(x) .
$$

Hence from $\lim _{k \rightarrow \infty} F\left(x+b_{k}\right)=1$, (6.1) follows.

Conditions $\left({ }^{* *}\right)$ and $\left({ }^{* *}\right)$ imply, by Gnedenko's theorem, that for all $x$

$$
\lim _{k \rightarrow \infty} \frac{\lg F\left(x+\beta_{k+1}\right)}{\lg F\left(\beta_{k}\right)}=\frac{\lg \Phi\left(x+b_{0}+\xi\right)}{\lg \Phi(\xi)}
$$

In particular, if $x=0$ we get, using (2.10),

$$
\lim _{k \rightarrow \infty} \frac{\lg \mathscr{F}\left(\beta_{k+1}\right)}{\lg F\left(\beta_{k}\right)}=r_{0}
$$

which proves that the sequence $\left\{n_{k}\right\}$ thus constructed satisfies condition (5.1).

REMARK 6.1. In defining the sequence $\left\{\beta_{k}\right\}$ it is possible to consider the distribution $\Phi(x / a)$ where $a>0$ and thus to get necessary and sufficient conditions so that $F^{n_{k}}\left(a x+b_{k}\right) \rightarrow \Phi(x)$ as $k \rightarrow \infty$ and (5.1).

REMARK 6.2. The assumption of continuity of the d.f. $F(x)$ may not be essential in our proof. It seems to us that the lemmas proved are still valid, if instead of a root of (6.10) we take a number $x^{\prime}$ such that $\left\{f\left(x^{\prime}+0\right)-A\right\} \cdot\left\{f\left(x^{\prime}-0\right)-A\right\} \leqq 0$ and replace the maximum and minimum by the upper and lower bounds respectively. The one-sided limits $f(x+0)$ and $f(x-0)$ exist since $f(x)$ is of bounded variation.

EXAMPLE 6.1. Let $\Phi(x)=\exp \left(-e^{-x+\cos x}\right)$. By Example 4.2, $\Phi(x) \in \Lambda^{*}$. Here $r_{0}=\exp (-2 \pi), b_{0}=2 \pi$. Let

$$
\mathscr{F}(x)= \begin{cases}0 & \text { if } x \leqq 0, \\ \exp \left(-e^{-x+\cos x-\lg x}\right) & \text { if } x>0 .\end{cases}
$$


If $b_{k}=2 k \pi, n_{k}=[2 \pi k \cdot \exp (2 \pi k)]$ it can be shown that conditions (5.1) and (6.1) are satisfied.

On the other hand if $\eta=\pi$ we have $\phi(x)=\exp (-\pi-2 \cos x)$ and $m=\exp (-\pi-2), \quad M=\exp (-\pi+2)$. Let $A=\exp (-\pi)$. Then equation (6.5) takes the form $\cos x=0$ and it has two d-roots in $[0,2 \pi): \xi_{1}=\pi / 2$ and $\xi_{2}=3 \pi / 2$. Equation (6.10) then has the form

$$
\cos x+(1 / 2) \lg (1+\pi / x)=0 .
$$

Let $t(1)$ be the first positive root of this equation. Then, since $\lim _{x \rightarrow \infty}(1+\pi / x)=1$, the sequence $t(n)$ has the form $t(n)=(2 n-1) \pi / 2+\varepsilon_{n}$, where $\varepsilon_{n} \rightarrow 0$. For $\left\{\beta_{k}\right\}$ we can choose, for instance,

$$
\beta_{k}=t(2 k+2)=2 k \pi+3 \pi / 2+\varepsilon_{k}^{\prime},
$$

where $\varepsilon_{k}^{\prime} \rightarrow 0$. In this way the sequence $\left\{\beta_{k}\right\}$ defines the sequence $\left\{b_{k}\right\}$ asymptotically exactly up to the additive constant $3 \pi / 2$, which is one of the d-roots of equation (6.5), i.e. $\cos x=0$ in $[0,2 \pi)$.

7. The attraction domain of distributions from the classes $\Phi^{*}$ and $\Psi^{*}$. We shall show here that the problem of determining the $r$-attraction domain $(r>0)$ of the classes $\Phi^{*}$ and $\Psi^{*}$ can be reduced to the special case studied in the previous section.

Lemma 7.1. Let $\Phi(x)$ be a proper distribution of the class $\Phi^{*}$, whose "left end" (2.3) satisfies the condition

$$
x_{0}=0 .
$$

If (1.2) and (5.1) are satisfied then also

$$
\lim _{k \rightarrow \infty} F^{n_{k}}\left(a_{k} x\right)=\Phi(x)
$$

and, for every $x,(6.7)$ holds.

Proof. From (7.1) follows (2.5) with $a>1$. Hence, by Lemma 3.1,

$$
\lim _{k \rightarrow \infty}\left(a_{k+1} / a_{k}\right)=a, \quad \lim _{k \rightarrow \infty}\left(\left(b_{k+1}-b_{k}\right) / a_{k}\right)=0 .
$$

Let $\varepsilon$ and $q(\varepsilon>0,1 / a<q<1)$ be any numbers. By (7.3) there exists an integer $p$ so that, for every non-negative integer $m$,

$$
a_{p+m} / a_{p+m+1}<q, \quad\left(b_{p+m+1}-b_{p+m}\right) / a_{p+m}<\varepsilon .
$$

For every natural number $n$ we have

$$
\frac{b_{p+n}}{a_{p+n}}=\frac{b_{p}}{a_{p+n}}+\sum_{m=0}^{n-1} \frac{b_{p+m+1}-b_{p+m}}{a_{p+m}} \cdot \frac{a_{p+m}}{a_{p+n}},
$$


hence using (7.4)

$$
\left|\frac{b_{p+n}}{a_{p+n}}\right| \leqq\left|\frac{b_{p}}{a_{p+n}}\right|+\varepsilon \sum_{m=1}^{n} q^{m} .
$$

Now since by (7.3) $a_{k} \rightarrow \infty \quad(k \rightarrow \infty), 0<q<1$ and $\varepsilon$ is arbitrary then $\lim _{k \rightarrow \infty}\left(b_{k} / a_{k}\right)=0$. This proves, by Gnedenko's theorem, equality (7.2). In order to prove (6.7) let us suppose that $F(b)=1$ for some $b$. Since $a_{k} \rightarrow \infty$ we would have, for every $x>0$ and every large $n, F\left(a_{n} x\right) \geqq F(b)=1$. Thus, by (7.2), $\Phi(x)=1$ for $x>0$, which together with (7.1) contradicts the assumption that $\Phi(x)$ is a proper distribution.

LEMMA 7.2. Let $\Phi(x)$ be a proper distribution of the class $\Psi *$, whose "right end" (1.6) satisfies the condition

$$
y_{0}=0 \text {. }
$$

If (1.2) and (5.1) are satisfied then there exists a number b so that also

$$
\lim _{k \rightarrow \infty} F^{n_{k}}\left(a_{k} x+b\right)=\Phi(x)
$$

and the d.f. $F(x)$ satisfies:

$$
F(b-\varepsilon)<1
$$

for every $\varepsilon>0$, and

$$
F(b-0)=1 .
$$

Proof. From (7.5) follows (2.5), where $0<a<1$. Hence also (7.3). For every $\varepsilon$ and $q(\varepsilon>0, a<q<1)$ there exists an integer $p$ such that for every integer $k \geqq p$ and every integer $m \geqq 0$

$$
a_{k+m+1} / a_{k+m}<q, \quad\left(b_{k+m+1}-b_{k+m}\right) / a_{k+m}<\varepsilon, \quad a_{k}<\varepsilon .
$$

For every integer $n \geqq 0$ we have

$$
\frac{b_{k+n}-b_{k}}{a_{k}}=\sum_{m=0}^{n-1} \frac{b_{k+m+1}-b_{k+m}}{a_{k+m}} \cdot \frac{a_{k+m}}{a_{k}} .
$$

Hence by (7.9)

$$
\left|b_{k+n}-b_{k}\right| / a_{k} \leqq \varepsilon \sum_{m=0}^{n-1} q^{m}<\varepsilon /(1-q)
$$

Therefore since $a_{k}<\varepsilon$, also $\left|b_{k+n}-b_{k}\right|<\varepsilon^{2} /(1-q)$. Thus the sequence $\left\{b_{k}\right\}$ converges. Let

$$
\lim _{k \rightarrow \infty} b_{k}=b
$$


By taking limits in (7.10) as $n \rightarrow \infty$ with fixed $k$ we obtain $\left|b-b_{k}\right| / a_{k} \leqq \varepsilon /(1-q)$. Hence, by Gnedenko's theorem, (7.6) follows.

Now suppose that for some $\varepsilon>0$

$$
F(b-\varepsilon)=1,
$$

where $b$ is the limit (7.11). Let $x$ be any number. Since $a_{k} \rightarrow 0$ then by (7.11), for large $k, a_{k} x+b_{k}>b-\varepsilon$. Thus, by (7.12), $F^{n_{k}}\left(a_{k} x\right)=1$ and, by (1.2), $\Phi(x) \equiv 1$, which is a contradiction proving (7.7).

From (1.2) follows that for every $x$

$$
\lim _{k \rightarrow \infty} F\left(a_{k} x+b_{k}\right)=1 \text {. }
$$

On the other hand for every $\varepsilon>0, a_{k} x+b_{k}<b+\varepsilon$ for large $k$. Thus for every $\varepsilon>0$

$$
F(b+\varepsilon)=1 .
$$

Now note that for every $x<0$

$$
a_{k} x+b_{k}<b
$$

from some $k$ on. Indeed, assume that for a certain $\xi<0$ and a subsequence $k_{s}$ we have $a_{k_{s}} \xi+b_{k_{s}} \geqq b$. Let $\eta(\xi<\eta<0)$ be any continuity point of the distribution $\Phi(x)$. Then also $a_{k_{s}} \eta+b_{k_{s}}>b$, hence, by (7.14), $F\left(a_{k_{s}} \eta+b_{k_{s}}\right)=1$ for every $s$. According to (1.2) this means that $\Phi(\eta)=1$, which contradicts (7.5).

Finally, since $\left(a_{k} x+b_{k}\right) \rightarrow b$ as $k \rightarrow \infty$, equality (7.8) follows from (7.13) and (7.15).

THEOREM 7.1. Let $\Phi(x)$ be a proper distribution of the class $\Phi^{*}$, respectively $\Psi^{*}$, let $r_{0}\left(0<r_{0}<1\right)$ be its maximal rank.

In order that $F(x)$ be attracted with a positive rank to $\Phi(x)$ the following conditions are necessary and sufficient:

$\left(^{*}\right)$ Condition (6.7), respectively, conditions (7.7) and (7.8).

$\left.{ }^{* *}\right)$ There exist real numbers $\beta_{k}$ and integers $n_{k}$, for which condition (5.1) holds, so that

$$
\lim _{k \rightarrow \infty} F^{n_{k}}\left(x+\beta_{k}\right)=\bar{\Phi}(x),
$$

where the distributions $F(x)$ and $\bar{\Phi}(x)$ are defined by

$$
F(x)=\left\{\begin{array}{ll}
0 & \text { if } x \leqq 0, \\
F\left(e^{x}\right) & \text { if } x>0,
\end{array} \quad \bar{\Phi}(x)=\Phi\left(x_{0}+e^{x}\right),\right.
$$

respectively,

$$
F(x)=F\left(b-e^{-x}\right), \quad \bar{\Phi}(x)=\Phi\left(y_{0}-e^{-x}\right) .
$$


Proof. I. Necessity. Let us assume that $F(x)$ is partially attracted with positive rank to $\Phi(x)$ in $\Phi^{*}$, and hence (1.2) and (5.1) hold. Therefore also

$$
\lim _{k \rightarrow \infty} F^{n_{k}}\left(a_{k} x+\bar{b}_{k}\right)=\Phi\left(x+x_{0}\right)
$$

where $\bar{b}_{k}=a_{k} x_{0}+b_{k}$. The distribution $\Phi\left(x+x_{0}\right)$ also satisfies condition (7.1) and Lemma 7.1 is applicable. Thus follows the necessity of $\left(^{*}\right)$. As was shown, (7.2) holds in our case. Let us substitute in (7.2) $e^{x}$ for $x>0$ and $\beta_{k}=\lg a_{k}$, then we obtain

$$
\lim _{k \rightarrow \infty} F^{n_{k}}\left(e^{x+\beta_{k}}\right)=\bar{\Phi}(x)
$$

It was shown in the proof of Lemma 7.1 that $a_{k} \rightarrow \infty$ as $k \rightarrow \infty$. Thus, for every $x, x+\beta_{k}>0$ for large $k$, hence also for sufficiently large $k$

$$
F\left(x+\beta_{k}\right)=F\left(e^{x+\beta_{k}}\right) .
$$

This and (7.17) imply the necessity of condition $\left({ }^{* *}\right)$. Note that by $\left({ }^{*}\right) F(x)$ is a distribution.

II. Sufficiency. Assume conditions $\left(^{*}\right)$ and $\left({ }^{* *}\right)$. By Theorem 2.3 the distribution $\bar{\Phi}(x)$ belongs to $\Lambda^{*}$. We have seen that in this case $\beta_{k} \rightarrow \infty(k \rightarrow \infty)$. Put in (7.16) $\lg x$ instead of $x$ and $a_{k}=\exp \left(b_{k}\right)$, then we get

$$
\lim _{k \rightarrow \infty} F^{n_{k}}\left(\lg a_{k} x\right)=\Phi\left(x+x_{0}\right)
$$

for $x>0$. By the definition of function $F(x)$

$$
F(x)=F(\lg x) \quad \text { for } x>1 .
$$

Since $a_{k} \rightarrow \infty$, for every $x>0$ and large $k, a_{k} x>1$ thus also $F\left(a_{k} x\right)=F\left(\lg a_{k} x\right)$ and by (7.18)

$$
\lim _{k \rightarrow \infty} F^{n_{k}}\left(a_{k} x\right)=\Phi\left(x+x_{0}\right)
$$

for $x>0$. Now, since $F(x)$ is a d.f., then clearly also

$$
\lim _{k \rightarrow \infty} F^{n_{k}}\left(a_{k} x\right)=0
$$

for $x<0$. Condition (*) implies that this holds also for $x=0$. On the other hand also $\Phi\left(x+x_{0}\right)=0$ for $x \leqq 0$. Thus equality (7.19) is fulfilled for every $x$.

Since, by assumption, (5.1) holds, the d.f. $F(x)$ is partially attracted to $\Phi(x)$ with positive rank.

The statement for $\Phi(x) \in \Psi^{*}$ is proved in a similar way. Here we use Lemma 7.2, and, by (7.7) and (7.8), $F(x)$ is a d.f. 
Acknowledgement. I wish to express my thanks to Professor A. Dvoretzky of the Hebrew University of Jerusalem for reading the manuscript and for his helpful comments.

\section{REFERENCES}

1. M. Fréchet, Sur la loi de probabilité de l'écart maximum, Ann. Soc. Polon. Math. Cracovie 6 (1927), 93.

2. B. Gnedenko, Sur la distribution limite du terme maximum d'une série aléatoire, Ann. of Math. (2) 44 (1943), 423-453.

3. B. V. Gnedenko and A. N. Kolmogorov, Limit distributions for sums of independent random variables, Addison-Wesley, Reading, Mass., 1954.

4. M. L. Juncosa, The asymptotic behavior of the minimum in a sequence of random variables, Duke Math. J. 16 (1949), 609-618.

5. D. G. Mejzler, On a problem of B. V. Gnedenko, Ukrain. Mat. Ž. 1(1949), no. 2, 67-84. (Russian)

6. - - On the limit distribution of the maximal term of a variational series, Dopovidi Akad. Nauk Ukraïn. RSR No. 1 (1950), 3-10. (Ukrainian. Russian Summary)

7. - _ The study of the limit laws for the variational series, Trudy Inst. Mat. Meh. Akad. Nauk Uzbek. SSR, No. 10 (1953), 96-105. (Russian)

8. - On the problem of the limit distributions for the maximal term of a variational series, L'vov. Politehn. Inst. Naučn. Zap. Ser. Fiz.-Mat. 38 (1956), no. 2, 90-109. (Russian)

9. - - On partial limit distributions for the maximal term of a variational series, L'vov. Politehn. Inst. Naučn. Zap. Ser. Fiz.-Mat. 30 (1955), no. 1, 24-44. (Russian)

10. A. Ja. Khintchine, Limit distributions for sums of independent random variables, ONTI, 1938. (Russian)

11. D. G. Mejzler, On a theorem of B. V. Gnedenko, Sb. Trudov Inst. Mat. Akad. Nauk Ukrain. RSR No. 12 (1949), 31-35. (Russian)

12. I. P. Natanson, Theory of functions of a real variable, Ungar, New York, 1955.

THE Hebrew UNIVERSITY, JERUSALEM, ISRAEL 